\title{
Current Trends in the Algebraic Image Analysis: A Survey
}

\author{
Igor Gurevich, Yulia Trusova, and Vera Yashina \\ Dorodnicyn Computing Centre, Russian Academy of Sciences, \\ Moscow, The Russian Federation \\ \{igourevi,ytrusova\}@ccas.ru, werayashina@gmail.com
}

\begin{abstract}
Survey. The main goal of the Algebraic Approach is the design of a unified scheme for the representation of objects for the purposes of their recognition and the transformation of such representations in the suitable algebraic structures. It makes possible to develop corresponding regular structures ready for analysis by algebraic, geometrical and topological techniques. Development of this line of image analysis and pattern recognition is of crucial importance for automated image mining and application problems solving. It is selected and briefly characterized main aspects of current state of the image analysis algebraization. Special attention is paid to the recent results of the Russian mathematical school.
\end{abstract}

Keywords: Image analysis, image algebras, descriptive approach, pattern recognition, image representations.

\section{Introduction}

Automation of image processing, analysis, estimating and understanding is one of the crucial points of theoretical computer science having decisive importance for applications.

The specificity, complexity and difficulties of image analysis and estimation (IAE) problems stem from necessity to achieve some balance between such highly contradictory factors as goals and tasks of a problem solving, the nature of visual perception, ways and means of an image acquisition, formation, reproduction and rendering, and mathematical, computational and technological means allowable for the IAE.

The mathematical theory of image analysis is not finished and is passing through a developing stage. It is only recently came understanding that only intensive creating of comprehensive mathematical theory of image analysis and recognition (in addition to the mathematical theory of pattern recognition) could bring a real opportunity to solve efficiently application problems via extracting from images the information necessary for intellectual decision making.

A new approach to analyzing and estimating information represented in the form of images - the Descriptive Approach to Image Analysis and Understanding (DA) by I.B.Gurevich [2, 6, 14-16] is based on the specialization of the "Algebraic Approach to Pattern Recognition and Classification Problems Solving” by Yu. I. Zhuravlev [43] for the case when the initial information is represented in the form of images. 


\section{State of the Art of the Mathematical Theory of Image Analysis}

To automate image mining, we need an integrated approach to implement the potential of mathematical apparatus being used in transforming and analyzing information represented in the form of images.

Image mining now tends to multiplicity (multi-algorithmic and multi-model modes) and to fusion of the results. It means that several different algorithms are applied in parallel to process the same model and several different models of the same initial data are used to solve the problem and then the results are fused to obtain the most accurate solution. Yu.I.Zhuravlev obtained the first and fundamental results in this area in 1970s [43].

From 1990s, the most part of pattern recognition applications and considerable part of research in artificial intelligence deal with images. As a result, new technical tools emerged to obtain information that allow representing of recorded and accumulated data in the form of images and the image recognition itself became more popular as the powerful and efficient methodology to process and analyze data mathematically and to detect hidden regularities.

There are a lot of internal scientific problems that have arisen within image recognition. First of all, these imply algebraizing the image recognition theory, arranging image recognition algorithms, estimating the algorithmic complexity of the image recognition problem, automating the synthesis of the corresponding efficient procedures, formalizing the description of the image as a recognition object, etc. These problems form the base of the mathematical agenda of the descriptive theory of image recognition developed using the ideas of the Algebraic Approach to Pattern Recognition and Classification.

There are three main issues one needs to overcome when dealing with images: 1) to describe images; 2) to develop, study and optimize the selection of mathematical methods and tools for data processing under image recognition; 3) to implement mathematical methods of image analysis via software and hardware means.

\section{$3 \quad$ Algebraization of Pattern Recognition and Image Analysis}

This section contains steps of the algebraization in image analysis fundamentals and the basic theories of pattern recognition, image algebras.

By now, image analysis and evaluation have a wide experience gained in applying mathematical methods from different sections of mathematics, computer science and physics, in particular algebra, geometry, discrete mathematics, mathematical logic, probability theory, mathematical statistics, mathematical analysis, mathematical theory of pattern recognition, digital signal processing, and optics.

However we still need a regular basis to arrange and choose suitable methods of image analysis, represent in an unified way the processed data (images), construct mathematical models of images designed for recognition problems, and, on the whole, to introduce the universal language for unified description of images and transformations over them. 
The necessity to solve complex recognition and specialized image languages generated attention to formal descriptions-models of initial data and formalization of descriptions of procedures of their transformation in the area of pattern recognition (and especially in image recognition in 1960s). As the substantial achievements in this "descriptive" line of R\&D we should mention publications by A. Rosenfeld [34], T. Evans [12], R. Narasimhan [29], R. Kirsh [21], A. Shaw [37], H. Barrow, A. Ambler, and R. Burstall [1], S. Kaneff [20].

In 1970s Yu.I.Zhuravlev proposed "The Algebraic Approach to Recognition and Classification Problems" [43], where he defined formalization methods for describing heuristic algorithms of pattern recognition and proposed the universal structure of recognition algorithms. In the same years, U. Grenander stated his "Pattern Theory" [18], where he considered methods of data representation and transformation in recognition problems in terms of regular combinatorial structures, leveraging algebraic and probabilistic apparatus. M.Pavel [31] introduced "Theory of Categories Techniques in Pattern Recognition", to describe pattern recognition algorithms via transforms of initial data preserving its class membership.

The Russian mathematical school also has important original results on algebraic tools for pattern recognition and image analysis in. There are algebras on algorithms, algebraic multiple classifiers, algebraic committees of algorithms, combinatorial algorithms for recognition of 2-D data [1], descriptive image models, 2-D formal grammars [34].

In the framework of scientific school of Yu.I.Zhuravlev several essential results were obtained by V.L.Matrosov [26], K.V.Rudakov [35] and V.D.Mazurov [27]. There are significant number of results concerned with algebraic methods of analysis and estimation of information represented as signals (G.Labunec [22], Ya.A.Furman [13], V.M.Chernov [4]).

Algebraization of pattern recognition and image analysis has attracted and continues to attract the attention of many researchers. First of all - the development of algebraic construction for image analysis and processing - formal grammars, cellular automata, mathematical morphology, image algebras, multiple algorithms, descriptive approach.

The idea of constructing a unified language for concepts and operations used in image processing appeared for the first time in works of Unger [42], who suggested to parallelize algorithms for processing and image analysis on computers with cellular architecture.

Mathematical morphology by G. Matheron [25] and J.Serra [36] became a starting point for a new mathematical wave in image analysis. Serra and Sternberg [39] were the first to succeed in constructing an integrated algebraic theory of processing and image analysis on the basis of mathematical morphology. It is believed [28] that it was precisely Sternberg who introduced the term "image algebra" in the current standard sense. The final version of image algebras (IA) was Standard Image Algebra by G.Ritter [32] (algebraic presentation of image analysis and processing operations).

Descriptive Image Algebras (DIA) is created as a new IA provided possibility to operate with main image models and with basic models of procedure of transforms, which lead to effective synthesis and realization of basic procedures of formal image description, processing, analysis and recognition. DIA is introduced by I.B.Gurevich and developed by him and his pupils I.V.Koryabkina, I.A.Jernova, A.A.Myagkov, A.A.Nefedov, Y.O.Trusova, V.V.Yashina [14-16]. 
In the history of algebraization we should mention: J.von Neumann [30], S.Unger [42] (studies of interactive image transformations in cellular space); M. Duff, D. Watson, T. Fountain, and G. Shaw [10] (a cellular logic array for image Processing); A. Rosenfeld [33] (digital topology); H.Minkowski and H.Hadwiger (pixel neighborhood arithmetic and mathematical morphology); G.Matheron, J.Serra, S.Sternberg [25, 36, 39] (a coherent algebraic theory specifically designed for image processing and image analysis - mathematical morphology); S. Sternberg [39] (the first to use the term "image algebra"); P. Maragos [24] (introduced a new theory unifying a large class of linear and nonlinear systems under the theory of mathematical morphology); L. Davidson [9] (completed the mathematical foundation of mathematical morphology by formulating its embedding into the lattice algebra known as Mini-Max algebra); G.Ritter [32] (Image Algebra); I.B.Gurevich [15] (Descriptive Image Algebra); T.R. Crimmins and W.M. Brown, R.M. Haralick, L. Shapiro, R.W. Schafer, J. Goutsias, L. Koskinen and Jaako Astola, E.R. Dougherty, P.D. Gader, M.A. Khabou, A. Koldobsky, B. Radunacu, M.Grana, F.X. Albizuri, P. Sussner [7,8,10,11,19,40] (recent papers on mathematical morphology and image algebras).

\section{Descriptive Approach to Image Analysis and Understanding}

This section contains a brief description of the principal features of the DA needed to understand the meaning of the introduction of the conceptual apparatus and schemes of synthesis of image models proposed to formalize and systematize the methods and forms of image representation.

By the middle of 1990s, it became obvious that for the development of image analysis and recognition, it is critical to: 1) understand the nature of the initial information - images, 2) find methods of image representation and description that allow constructing image models designed for recognition problems, 3) establish the mathematical language designed for unified description of image models and their transformations that allow constructing image models and solving recognition problems; 4) construct models to solve recognition problems in the form of standard algorithmic schemes that allow, in the general case, moving from the initial image to its model and from the model to the sought solution.

The DA gives an unified conceptual structure that helps to develop and implement these models and the mathematical language [14-17]. The main DA purpose is to structure and standardize different methods, operations and representations used in image recognition and analysis. The DA provides the conceptual and mathematical basis for image mining, with its axiomatic and formal configurations giving the ways and tools to represent and describe images to be analyzed and evaluated.

Experience in the development of the mathematical theory of image analysis and its use to solve applied problems shows that, when working with images, it is necessary to solve problems that arise in connection with the three basic issues of image analysis: 1) the description of images; 2) the development, exploration, and optimization of the selection of mathematical methods and tools for information processing in the analysis of images; 3) the hardware and software implementation of the mathematical methods of image analysis. 
Mathematical foundations of the DA are as follows: 1) the algebraization of the extraction of information from images; 2) the specialization of the Zhuravlev algebra to the case of representation of recognition source data in the form of images; 3) a standard language for describing the procedures of the analysis and recognition of images (DIA) [14-16]; 4) the mathematical formulation of the problem of image recognition; 5) mathematical theories of image analysis and pattern recognition; 6) a model of the process for solving a standard problem of image recognition. The main objects and means of the DA are: 1) images; 2) a universal language (DIA); 3 ) 2 types of descriptive models: a) an image model; b) a model for solving procedures of problems of image recognition and their implementation; 4) descriptive algebraic schemes of image representation (DASIR); 5) multi-model and multi-aspect representations of images, which are based on generating descriptive trees (GDT) [14-16].

The basic methodological principles of the DA are: 1) the algebraization of the image analysis; 2) the standardization of the representation of problems of analysis and recognition of images; 3 ) the conceptualization and formalization of phases through which the image passes during transformation while the recognition problem is solved; 4) the classification and specification of admissible models of images (descriptive image model - DIM); 5) RIRF; 6) the use of the standard algebraic language of DIA for describing models of images and procedures for their construction and transformation;7) the combination of algorithms in the multi-algorithmic schemes; 8) the use of multi-model and multi-aspect representations of images; 9) the construction and use of a basic model of the solution process for the standard problem of image recognition; 10) the definition and use of non-classical mathematical theory for the recognition of new formulations of problems of analyzing and recognizing images.

\section{$5 \quad$ Ontology-Based Approach to Image Analysis}

The automation of image analysis assumes that researchers and users of different qualifications have at their disposal not only a standardized technology of automation, but also a system supporting this technology, which accumulates and uses knowledge on image processing, analysis and evaluation and provides adequate structural and functional possibilities for supporting the more intelligent choice and synthesis of methods and algorithms. The automated system (AS) for image analysis must combine the possibilities of the instrumental environment for image processing and analysis and a knowledge-based system. Therefore, one of its main components is a knowledge base. Knowledge bases usually contain modules of universal knowledge, which are not related to any subject domain and knowledge modules related to a certain subject domain. The AS must provide software implementation of the hierarchies of classes of the main objects used in image analysis, have a specialized user interface, contain a library of algorithms that allow one to solve the main problems of image analysis and understanding with the help of efficient computational procedures, and provide accumulation and structuring of knowledge and experience in the domain of image analysis and understanding.

The need of efficient knowledge representation facilities can be fulfilled by using a suite of ontologies, For example, in [23], an approach devoted to semantic image 
interpretation for complex object classification purposes is proposed. The work described in [5] addresses the problem of explicit representation of objectives when developing image processing applications. The proposed framework demonstrates that ontology-based content representation can be used as an effective way for hierarchical and goal-directed inference in high-level visual analysis tasks.

In [6], a novel knowledge-oriented approach to image analysis based on the use of thesauruses and ontologies as tools for representation of knowledge, which are necessary for making intelligent decisions on the basis of information extracted from images, is proposed. The main contribution of this work is the development of a sufficiently detailed and well-structured Image Analysis Ontology. As a main source of the information about concepts the Image Analysis Thesaurus (IAT) [2] has been used. The important feature of the IAT is a novel hierarchical classification of tasks and algorithms for image processing, analysis and recognition.

\section{Conclusions}

The main deduction from the analysis of current trends in mathematical image analysis is necessity of a new algebraic language for describing and representing of image processing and understanding procedures and models. The new image algebra has to make possible: a) processing of images as objects of analysis and recognition; b) operations on image models and on models of procedures for image transformations; c) implementation of the procedures for image modifications both as operations and operands of the new image algebras for construction of compositions of basic models of procedures. The future research will be mostly devoted to constructing of image formal descriptions, i.e. to the Image Formalization Space and, in particular, to a) its topological properties; b) embedding of descriptive algorithmic schemes for image analysis into this space.

Acknowledgments. This work was supported in part by the Russian Foundation for Basic Research (projects Nos. 11-01-00990, 12-07-31123) and by the Presidium of the Russian Academy of Sciences within the Program "Information, Control, and Intelligent Technologies and Systems" (project No. 204) and the Program of the Division of Mathematical Sciences, of the Russian Academy of Sciences "Algebraic and Combinatorial Methods of New Generation Mathematical Cybernetics and Information Systems".

\section{References}

1. Barrow, H.G., Ambler, A.P., Burstall, R.M.: Some Techniques for Recognizing Structures in Pictures. In: Watanabe, S. (ed.) Proceedings of the International Conference on Frontiers of Pattern Recognition, pp. 1-30. Academic Press (1972)

2. Beloozerov, V.N., Gurevich, I.B., Gurevich, N.G., Murashov, D.M., Trusova, Y.O.: Thesaurus for Image Analysis: Basic Version. In: Pattern Recognition and Image Analysis: Advances in Mathematical Theory and Applications, vol. 13(4), pp. 556-569. Pleiades Publishing, Inc. (2003) 
3. Bloehdorn, S., et al.: Semantic Annotation of Images and Videos for Multimedia Analysis. In: Gómez-Pérez, A., Euzenat, J. (eds.) ESWC 2005. LNCS, vol. 3532, pp. 592-607. Springer, Heidelberg (2005)

4. Chernov, V.M.: Clifford Algebras Are Group Algebras Projections. In: Bayro-Corrochano, E., Sobczyk, G. (eds.) Advances in Geometric Algebra with Applications in Science and Engineering, pp. 467-482. Birkhauser, Boston (2001)

5. Clouard, R., Renouf, A., Revenu, M.: An Ontology-Based Model for Representing Image Processing Application Objectives. International Journal of Pattern Recognition and Artificial Intelligence 24(8), 1181-1208 (2010)

6. Colantonio, S., Gurevich, I., Pieri, G., Salvetti, O., Trusova, Y.: Ontology-Based Framework to Image Mining. In: Gurevich, I., Niemann, H., Salvetti, O. (eds.) Image Mining Theory and Applications: Proceedings of the 2nd International Workshop on Image Mining Theory and Applications (in conjunction with VISIGRAPP 2009), pp. 11-19. INSTICC Press, Lisboa (2009)

7. Crespo, J., Serra, J., Schaffer, R.W.: Graph-based Morphological Filtering and Segmentation. In: Proc. 6th Symp. Pattern Recognition and Image Analysis, Cordoba, pp. 80-87 (1995)

8. Crimmins, T., Brown, W.: Image Algebra and Automatic Shape Recognition. IEEE Transactions on Aerospace and Electronic Systems 21(1), 60-69 (1985)

9. Davidson, J.L.: Classification of Lattice Transformations in Image Processing. Computer Vision, Graphics, and Image Processing: Image Understanding 57(3), 283-306 (1993)

10. Duff, M.J.B., Watson, D.M., Fountain, T.J., Shaw, G.K.: A Cellular Logic Array for Image Processing. Pattern Recognition 5(3), 229-247 (1973)

11. Dougherty, E.R.: A Homogeneous Unification of Image Algebra. Part I: The Homogenous Algebra, part II: Unification of Image Algebra. Imaging Science 33(4), 136-143, 144-149 (1989)

12. Evans, T.G.: Descriptive Pattern Analysis Techniques: Potentialities and Problems. In: The Proceedings of the International Conference on Methodologies of Pattern Recognition, pp. 149-157. Academic Press (1969)

13. Furman, Y.A.: Parallel Recognition of Different Classes of Patterns. Pattern Recognition and Image Analysis 19(3), 380-393 (2009)

14. Gurevich, I.B., Yashina, V.V.: Operations of Descriptive Image Algebras with One Ring. Pattern Recognition and Image Analysis: Advances in Mathematical Theory and Applications 16(3), 298-328 (2006)

15. Gurevich, I.B., Yashina, V.V.: Computer-Aided Image Analysis Based on the Concepts of Invariance and Equivalence. Pattern Recognition and Image Analysis: Advances in Mathematical Theory and Applications 16(4), 564-589 (2006)

16. Gurevich, I.B., Yashina, V.V.: Descriptive Approach to Image Analysis: Image Formalization Space. Pattern Recognition and Image Analysis: Advances in Mathematical Theory and Applications 22(4), 495-518 (2012)

17. Gader, P.D., Khabou, M.A., Koldobsky, A.: Morphological Regularization Neural Networks. Pattern Recognition 33, 935-944 (2000)

18. Grenander, U.: Elements of Pattern Theory. The Johns Hopkins University Press (1996)

19. Haralick, R., Shapiro, L., Lee, J.: Morphological Edge Detection. IEEE J. Robotics and Automation RA-3(1), 142-157 (1987)

20. Kaneff, S.: Pattern Cognition and the Organization of Information. In: Watanabe, S. (ed.) The Proceedings of the International Conference on Frontiers of Pattern Recognition, pp. 193-222. Academic Press (1972) 
21. Kirsh, R.: Computer Interpretation of English Text and Picture Patterns. IEEE-TEC EC13(4) (1964)

22. Labunec, V.G.: Algebraic Theory of Signals and Systems (Digital Signal Processing). Krasnoyarsk University (1984)

23. Maillot, N., Thonnat, M., Boucher, A.: Towards ontology-based cognitive vision. Machine Vision and Applications 16, 33-40 (2004)

24. Maragos, P.: Algebraic and PDE Approaches for Lattice Scale-Spaces with Global Constraints. International Journal of Computer Vision 52(2/3), 121-137 (2003)

25. Matheron, G.: Random Sets and Integral Geometry. Wiley, New York (1975)

26. Matrosov, V.L.: The Capacity of Polynomial Expansions of a Set of Algorithms for Calculating Estimates. USSR, Comput. Maths. Math. Phys. 24(1), 79-87 (1985)

27. Mazurov, V.D., Khachai, M.Y.: Parallel Computations and Committee Constructions. Journal Automation and Remote Control 68(5), 912-921 (2007)

28. Miller, P.: Development of a Mathematical Structure for Image Processing: Optical division tech. report. Perkin-Elmer (1983)

29. Narasimhan, R.: Picture Languages. In: Kaneff, S. (ed.) Picture Language Machines, pp. 1-30. Academic Press (1970)

30. von Neumann, J.: The General Logical Theory of Automata. In: Celebral Mechenism in Behavior: The Hixon Symposium. John Wiley \& Sons (1951)

31. Pavel, M.: Fundamentals of Pattern Recognition. Marcell, Dekker, Inc., New York (1989)

32. Ritter, G.X.: Image Algebra. Center for computer vision and visualization, Department of Computer and Information science and Engineering, University of Florida, Gainesville, FL 32611 (2001)

33. Rosenfeld, A.: Digital Topology. American Math Monthly, 86 (1979)

34. Rosenfeld, A.: Picture Languages. Formal Models for Picture Recognition. Academic Press (1979)

35. Rudakov, K.V.: Universal and local constraints in the problem of correction of heuristic algorithms. Cybernetics 23(2), 181-186 (1987)

36. Serra, J.: Image Analysis and Mathematical Morphology. Academic Press (1982)

37. Shaw, A.: A Proposed Language for the Formal Description of Pictures. CGS Memo, 28, Stanford University (1967)

38. Schlesinger, M., Hlavac, V.: Ten Lectures on Statistical and Structural Pattern Recognition. In: Computational Imaging and Vision, vol. 24, 520 p. Kluwer Academic Publishers, Dordrecht (2002)

39. Sternberg, S.R.: Grayscale Morphology. Computer Vision, Graphics and Image Processing 35(3), 333-355 (1986)

40. Sussner, P.: Observations on Morphological Associative Memories and the Kernel Method. Neurocomputing 31, 167-183 (2000)

41. Town, C.: Ontological inference for image and video analysis. Machine Vision and Applications 17(2), 94-115 (2006)

42. Unger, S.H.: A Computer Oriented Toward Spatial Problems. Proceedings of the IRE 46, 1744-1750 (1958)

43. Zhuravlev, Y.I.: An Algebraic Approach to Recognition and Classification Problems. Pattern Recognition and Image Analysis: Advances in Mathematical Theory and Applications 8, 59-100 (1998) 